\title{
Plasma Temperature and Electron Density Determination Using Laser-Induced Breakdown Spectroscopy (LIBS) in Earth's and Mars's Atmospheres
}

\author{
Julian Stetzler, Shijun Tang and Rosemarie C. Chinni * \\ Department of Math and Science, Alvernia University, Reading, PA 19607, USA; JULIANPS@email.sc.edu (J.S.); \\ shijun.tang@alvernia.edu (S.T.) \\ * Correspondence: rosemarie.chinni@alvernia.edu; Tel.: +1-610-568-1492
}

Received: 4 August 2020; Accepted: 21 August 2020; Published: 25 August 2020

check for updates

\begin{abstract}
The purpose of this study was to calculate and compare the plasma temperatures and electron densities from the laser-induced breakdown spectroscopy (LIBS) data collected by NASA's Martian rover and compare them to samples measured in Earth's atmosphere. Using the Boltzmann plots, LIBS plasma temperatures were obtained for each site. The analysis focused on titanium lines that were located in the spectral region between 300 and $310 \mathrm{~nm}$. The electron density was measured using the Stark broadening of the hydrogen line at $656.6 \mathrm{~nm}$; the full width at half maximum (FWHM) of this line can be measured and correlated to the electron density of the plasma. Due to a neighboring carbon peak with the hydrogen line seen in many of the spectra from the Martian sites, the FWHM needed to be calculated using a computer program that completed the other side of the hydrogen line and then it calculated the FWHM for those data samples affected by this. The plasma temperatures and electron densities of the Martian sites were compared to LIBS samples taken on Earth.
\end{abstract}

Keywords: atomic spectroscopy; LIBS; plasma temperature; electron density

\section{Introduction}

The National Aeronautics and Space Administration (NASA) released over 300,000 data files from laser-induced breakdown spectroscopy (LIBS) studies conducted by the Mars Science Laboratory (MSL) Curiosity rover, which was launched in November of 2011. The ChemCam system on the Curiosity rover highlights many of the important applications of LIBS, and how LIBS is such a useful technique for analytical chemistry and planetary exploration. LIBS has been suggested as a useful technique for planetary exploration since 1990. The LIBS system aboard the MSL rover can provide elemental composition data on spots between 0.35 and $0.55 \mathrm{~mm}$ at distances over $7 \mathrm{~m}$. Combined with the remote microimager (RMI), an image of the sample, and laser spot can aid in applying context to the data, while also operating at the same range. The remote-sensing capabilities are not the only reason the LIBS ChemCam system was an excellent choice for the Martian mission. The many applications of LIBS in detecting trace elements from carbon, oxygen, nitrogen, to heavy metals such as lead are invaluable for achieving the scientific objectives of the MSL. The scientific objectives of the curiosity rover were to explore the history of organic and volatile chemicals. The elements of interest, $\mathrm{C}, \mathrm{H}, \mathrm{P}, \mathrm{Mn}, \mathrm{N}, \mathrm{O}$, etc., can be detected remotely with LIBS. Additionally, the small spot size of the LIBS system allows it to target specific layers in the sedimentary rocks. This precise study of the sediment can be used to better understand the geological history of Mars and the history of carbon concentrations on Mars. The LIBS system could also be used to study hydration states and help understand the current water cycle on Mars. Finally, the LIBS system could be used to detect various toxic elements, such as lead, 
arsenic, etc., and determine their concentrations in the dust on Mars to better understand the hazards of the Martian environment for human exploration. This illustrates some of the many applications of LIBS to the Martian mission and planetary exploration but also shows some of the many important applications of LIBS as an analytical technique [1-3].

These many advantages of LIBS (i.e., the ability to work on any sample, remote-sensing capability, rapid analysis, and virtually no sample preparation) make it invaluable for the Martian mission. The key component of using LIBS as an analytical technique is the spectrum emitted by the plasma. The plasma formed from LIBS, which is used for any quantitative or qualitative analysis of a sample, has been studied since the 1960s. Important plasma characteristics such as the plasma temperature and electron density on different surfaces and under different conditions have been analyzed by multiple studies. However, the Martian mission offers a new and exciting opportunity, to study and compare LIBS plasmas formed on an entirely new celestial body to those formed on Earth. The environment of Mars, especially its atmospheric pressure and composition and other parameters could potentially affect the characteristics of the LIBS plasma. Therefore, analyzing the data gathered by the NASA rover could provide valuable insight on how environmental factors influence the plasma generated by LIBS, thus leading to a better understanding of LIBS plasma characteristics and using it for quantitative and qualitative analysis [4-7].

The formation of the plasma is an extremely complicated process, one that could be better understood. The formation of the plasma occurs via inverse bremsstrahlung (free electron interactions) and involves collisions between photons (particles of light), electrons and atoms/molecules. The expansion, transferring of energy throughout the surface, and the time at which the plasma decays are all dependent on factors such as the physical state of the sample, and whether it is in a vacuum. Meanwhile, the energy levels and excitation of atoms within the sample which form the plasma are dependent on the thermodynamic equilibrium and interactions which fall under the broad category of matrix effects [4].

Thermodynamic equilibrium is one that is not well understood. In many cases, it is approximated to be local thermodynamic equilibrium. The concept of thermodynamic equilibrium would allow the temperature of the plasma to describe the many different properties of plasma such as electron densities. The plasma characteristics are dependent on factors such as atmospheric composition and pressure, laser intensity and spot size. Additionally, electron densities measured at significantly different atmospheric pressures will produce results that are orders of magnitude different. Thus, it is expected that the electron densities on Mars and likely the plasma temperatures will be significantly different due to the significantly different atmospheric conditions on Mars [4,6].

However, it is also important to understand that the formation of the plasma itself, and the many ways to characterize it, are greatly affected by other factors. As previously mentioned, the surrounding atmospheric gases and pressure can affect the characteristics of the plasma such as the temperature and electron densities that are being studied. However, other factors such as the incident laser intensity, spot size and even the distance from the target surface can affect the plasma characteristics as well $[1,3,8,9]$.

The Boltzmann plot method is a very common method for determining plasma temperatures. The Boltzmann plot method has proven to provide a precise average temperature of the plasma. It has proven more precise than alternatives such as the two-line Boltzmann method. Both methods compare the intensities of lines, but the two-line one compares the intensity ratios of only two lines to determine the plasma temperature, whereas the full Boltzmann plot does so graphically with multiple atomic emission lines [4,7].

Electron density is the number of free electrons, which are electrons separated from their atoms, per unit volume. Many studies use the well-established technique of Stark broadening to calculate electron densities, which has been shown to have good agreement between the experimental results and theoretical values. The line broadening in a LIBS plasma is mainly caused by Doppler width and the Stark effect. The Doppler width is dependent on the temperature and atomic mass of the emitting 
atoms. If only hydrogen lines are used for the electron density determination, the Doppler width can be disregarded, since it is only about $0.4-0.7 \mathrm{~nm}$ [8-10].

The Stark effect is a type of pressure broadening. In a plasma, this is attributed to interactions caused by the collisions of primarily ions but also electrons. The broadening of the hydrogen line is caused mostly by the Stark effect. As mentioned previously, some of the broadening can be attributed to the Doppler width, however, it is so minor that it is disregarded. Therefore, the electron density can be determined using the broadening of the Balmer-alpha hydrogen line due to the Stark effect $[8,11]$.

The purpose of this study was to analyze a subset of the Martian data for electron density and plasma temperature determination and then compare it to the data taken on Earth using similar laser energy parameters. The ChemCam data is publicly available [12].

\section{Results}

The Boltzmann plots for the Martian data produced correlation coefficients $\left(R^{2}\right)$ primarily above 0.80; a sample plot is shown in Figure 1. Table 1 shows the temperatures calculated for the Martian samples. The average temperature was found to be $18,900 \mathrm{~K}$. Please note only a small subset of the data is shown here; the average, standard deviation, and \%RSD (relative standard deviation) shown in the table is representative of all the temperatures calculated, which were 1184 temperatures in total. It is not known how the names of each site analyzed were determined; this information could not be found. It is known that the rover traveled and took its LIBS data within a 12 mile stretch of the Gale crater on Mars for the data used in this study. The Martian temperature data showed higher variation in the calculated plasma temperatures. This larger variation in plasma temperatures could be attributed to many different factors. Since the atmospheric pressure and composition are mostly consistent across Mars, and the Curiosity rover stayed within the Gale crater and did not travel a large distance between locations, it is unlikely that these factors caused the variations. Instead, it was likely other factors such as incident laser intensity, spot size and even the distance from the target surface were responsible for these variations in plasma temperatures [6]. In fact, given the large range of distances over which the LIBS system was used-up to $14 \mathrm{~m}$ [3]—it is possible that the variations in temperature and spectral intensity were caused by these different distances.

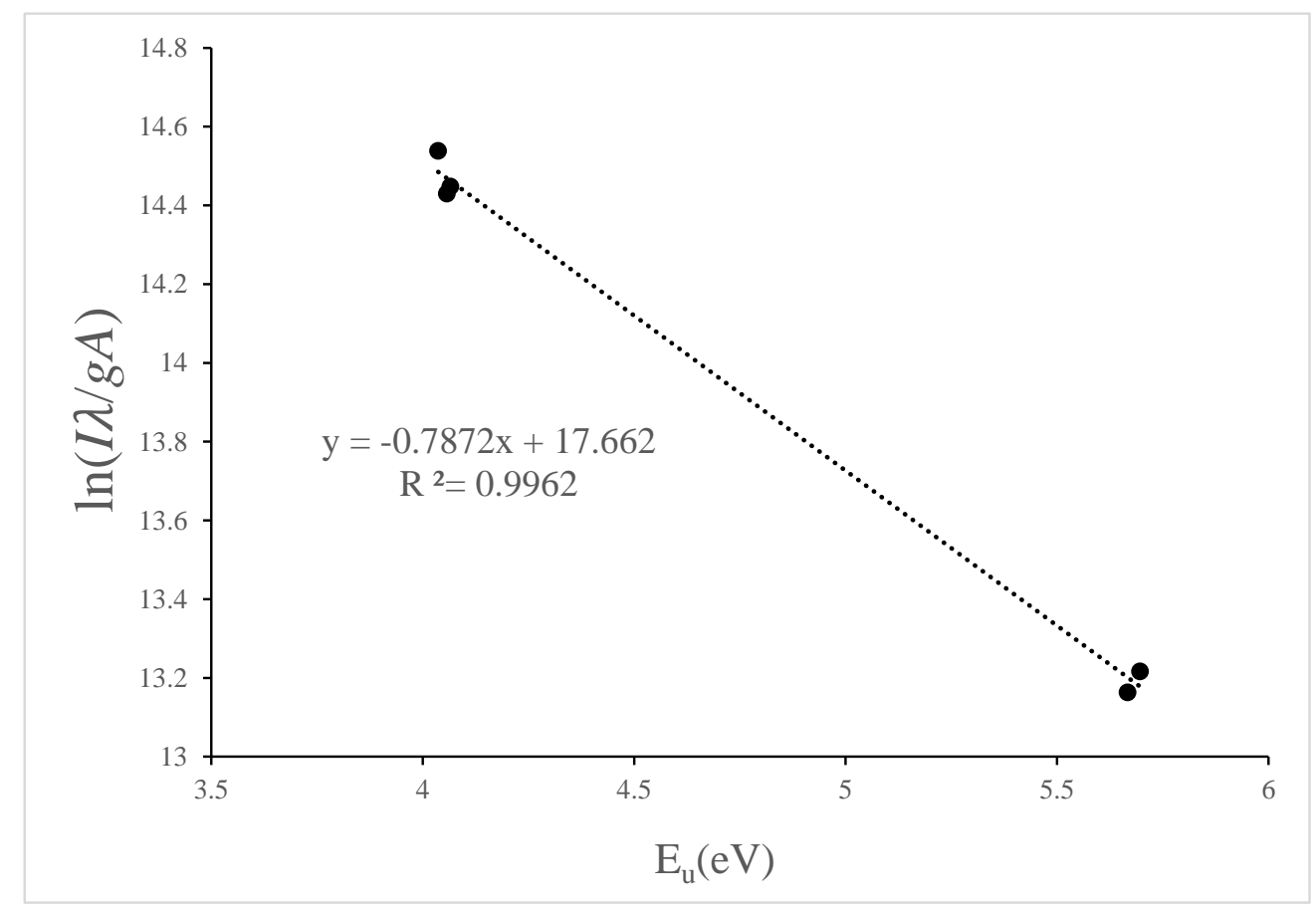

Figure 1. Boltzmann plot for location 1 labeled as Brazer from the Martian data. 
Table 1. Temperatures of a selection of Martian sites.

\begin{tabular}{|c|c|}
\hline Site & Temperature (K) \\
\hline Brazer & 14,700 \\
\hline Young_Point & 16,300 \\
\hline Reddick_Bight & 16,800 \\
\hline Rusty_Shale & 22,600 \\
\hline Whit_Cliffs_2 & 15,200 \\
\hline Cockscomb & 14,500 \\
\hline Platypus_Ridge & 19,900 \\
\hline Douglass_2 & 34,100 \\
\hline Airfield & 14,800 \\
\hline King_CCAM & 14,700 \\
\hline Peridido & 17,500 \\
\hline Lee_Flat & 13,200 \\
\hline Carrara & 20,000 \\
\hline CC_BT_0795a & 14,400 \\
\hline Aztec & 15,400 \\
\hline Uniab & 16,400 \\
\hline Tumas & 19,600 \\
\hline Hall_Canyon & 21,000 \\
\hline Keeler_Canyon & 16,600 \\
\hline Stephen_DP_2 & 29,300 \\
\hline Jarrad & 23,600 \\
\hline Cow_Bore & 23,000 \\
\hline Marienfluss & 16,900 \\
\hline Dunkirk & 15,800 \\
\hline Duperow & 19,400 \\
\hline Maywood & 16,900 \\
\hline Steep_Rock & 25,200 \\
\hline Camp_Island & 35,400 \\
\hline Big_Arm_2 & 14,600 \\
\hline King_Leopold & 19,300 \\
\hline Horseshoe_Hills & 12,400 \\
\hline Bonanza_King1_ccam & 15,400 \\
\hline Osiris & 17,200 \\
\hline Dwyka & 15,400 \\
\hline Crestaurum_1 & 15,200 \\
\hline CC_BT_0788a & 15,300 \\
\hline Saddle_Peak & 22,200 \\
\hline Ibex_Pass_785 & 15,000 \\
\hline Rove & 20,900 \\
\hline
\end{tabular}


Table 1. Cont.

\begin{tabular}{cc}
\hline Site & Temperature (K) \\
\hline Ramah & 23,600 \\
\hline Rocknest3 & 18,600 \\
\hline Cumberland3 & 19,900 \\
\hline Cumberland_Bowl & 16,900 \\
\hline Upheaval_Dome & 24,800 \\
\hline Mt_Tug & 16,600 \\
\hline CumberlandNew_RP & 18,100 \\
\hline Pearson & 21,300 \\
\hline Ghaub & 19,700 \\
\hline Gemsboktal & 20,400 \\
\hline CC_BT_0717a & 15,300 \\
\hline Chioak & 22,400 \\
\hline Saglek & 15,000 \\
\hline Gogebic & 23,200 \\
\hline Maddox & 17,000 \\
\hline Bwabata & 12,000 \\
\hline Average * & $\mathbf{1 8 , 9 0 0}$ \\
\hline Standard Deviation * & $\mathbf{4 6 4 0}$ \\
\hline \%RSD & $\mathbf{2 4 . 5}$
\end{tabular}

* Represents the average, standard deviation, and percent relative standard deviation of all of the temperatures calculated (1184 total).

It should be noted that about 280 LIBS Martian spectra could not be used for plasma temperature determination. This was due to the fact that these spectra did not contain any atomic lines or there were no titanium lines found in the region of interest. The actual cause of the unusable spectra could be attributed to multiple issues like the detector on the ChemCam not picking up any light or being too far away. According to NASA, the ChemCam device was effective at a range of $14 \mathrm{~m} \mathrm{[1-3].}$

The Boltzmann plots on the Earth samples showed correlations of generally above 0.78 . The average temperature was found to be $15,900 \mathrm{~K}$ in the $1 \mu$ s time delay data. Also, there was not as much variation in temperature as in the Martian data; these data are shown in Table 2. This lower variation in the Earth data is most likely due to the fact that the LIBS system parameters of laser energy, lens-to-sample distance, and placement of collection fiber were constant for this part of the experiment and/or there was a smaller data set.

Figure 2 shows a comparison between the average temperatures calculated from the Martian and Earth samples. The graph clearly shows that the temperatures calculated from the Earth samples were generally lower than the Martian samples. It also shows that the average temperatures were not significantly different from each other. It was thought that the potential cause for the lower plasma temperatures on Earth could be due to the fact that the data were taken in gated mode and the earliest part of the plasma formation where the plasma is hottest was not taken in account. However, these data were also taken using a zero-microsecond time delay with a $20 \mu$ s gate width; analysis of these data shows a slightly lower plasma temperature than the one-microsecond time delay; these data were added in Table 2 . The temperatures were lower using a time delay of $0 \mu \mathrm{s}$ but they were not significantly different from the $1 \mu$ s time delay temperature data. The average temperature here was determined to be $13,200 \mathrm{~K}$. 
Table 2. Plasma temperatures and electron densities of the earth samples.

\begin{tabular}{ccccc}
\hline Sample & $\begin{array}{c}\text { Temperature (K), } \\
\text { Data with } \mathbf{1} \boldsymbol{\mu} \mathbf{~ t d}\end{array}$ & $\begin{array}{c}\text { Temperature (K), } \\
\text { Data with } \mathbf{~} \boldsymbol{\mu s ~ t d}\end{array}$ & $\begin{array}{c}\text { Electron Density } \\
\left.\text { Using H } \mathbf{( c m}^{-3}\right)\end{array}$ & $\begin{array}{c}\text { Electron Density } \\
\text { Using Si }\left(\mathbf{c m}^{-3}\right)\end{array}$ \\
\hline black soil & 16,900 & 14,800 & $1.38 \times 10^{17}$ & $1.37 \times 10^{18}$ \\
\hline red soil & 14,300 & 12,400 & $1.10 \times 10^{17}$ & $1.56 \times 10^{18}$ \\
\hline synthetic silicate & 13,500 & 12,600 & $1.31 \times 10^{17}$ & $1.56 \times 10^{18}$ \\
\hline soil & 18,900 & 13,000 & $1.38 \times 10^{17}$ & $1.36 \times 10^{18}$ \\
\hline Average & 15,900 & 13,200 & $1.29 \times 10^{17}$ & $1.46 \times 10^{18}$ \\
\hline standard deviation & 2470 & 1080 & $1.29 \times 10^{16}$ & $1.14 \times 10^{17}$ \\
\hline \%RSD & 15.5 & 8.19 & 10.0 & 7.79 \\
\hline
\end{tabular}

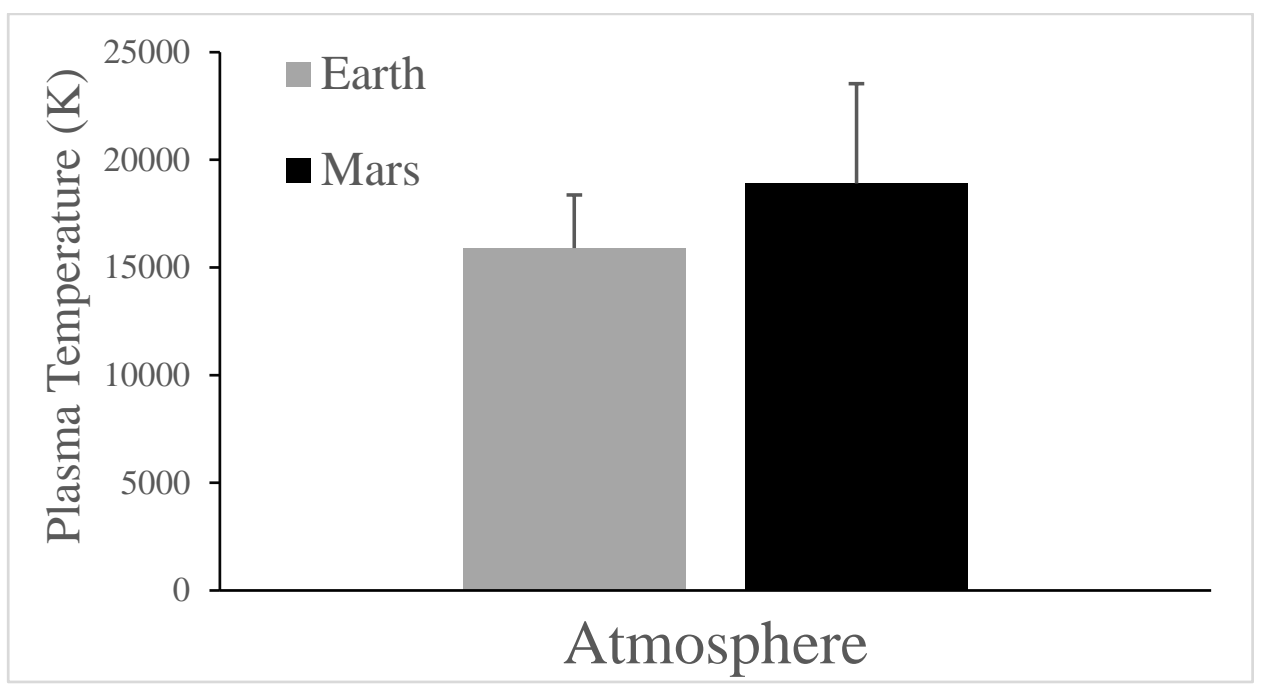

Figure 2. A comparison of the average plasma temperatures for Earth and Mars.

For the electron density determination of the Martian samples, the data files that produced no temperature results were neglected for this analysis. There were only about 80 samples that contained no carbon interference with the hydrogen line or for which the carbon peak did not interfere with the FWHM calculation. For the rest of the data, there was a large carbon line which interfered with the hydrogen line such that the FWHM could not be determined easily (Figure 3); this is due to the atmosphere of Mars, which is approximately $95 \% \mathrm{CO}_{2}$. For some of this data, it was possible to predict the other half of hydrogen peak to allow for calculation of its FWHM by using a computer program written. This allowed for electron density calculations for about 270 more samples. The electron density results showed slightly less variation than the temperature results; this is most likely due to the smaller subset of usable data. A smaller subset of the electron density data from the Martian data is shown in Table 3; the average electron density in these data was determined to be $2.17 \times 10^{17} \mathrm{~cm}^{-3}$. For the Earth samples, there was no carbon interference with the hydrogen line; therefore, the FWHM and electron densities were easy to determine. The electron density results for the Earth samples are shown in Table 2. 


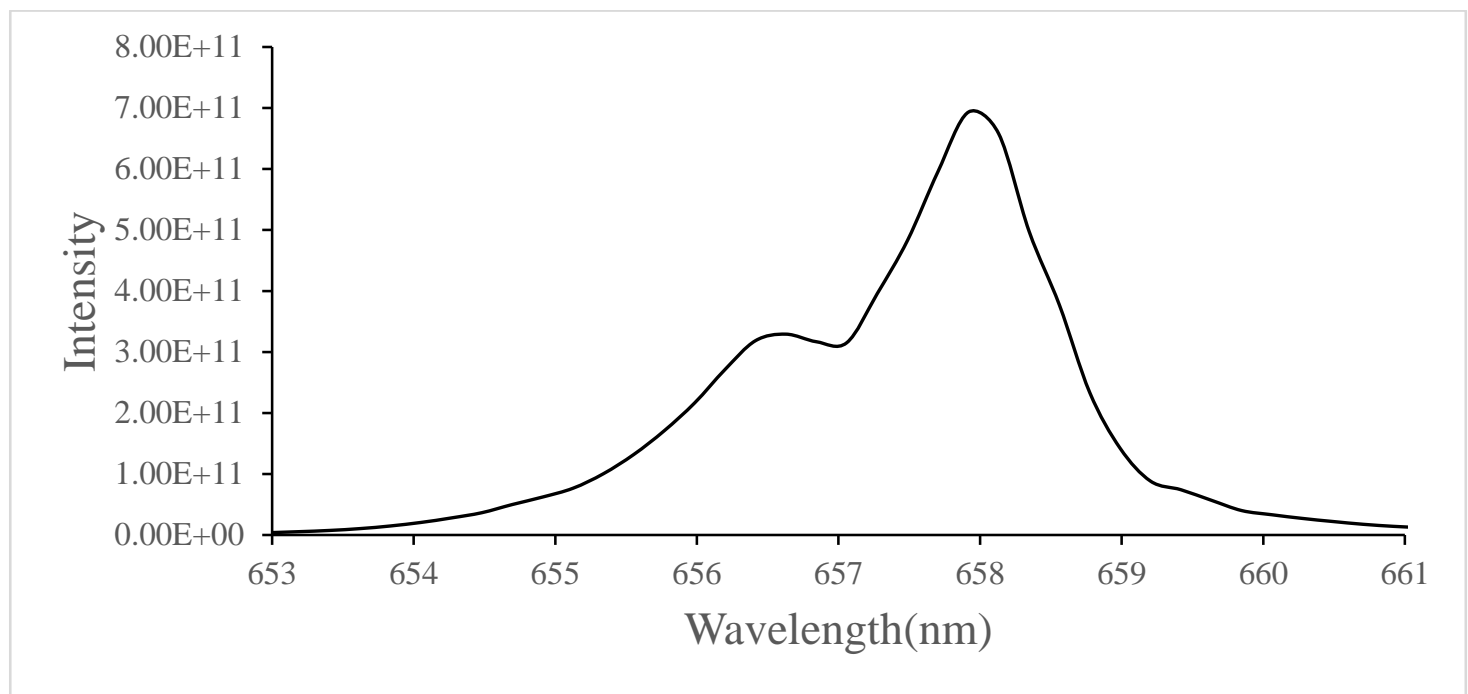

Figure 3. Spectral plot showing the carbon interference with the hydrogen peak. The spectrum is from location Aegis_post_1417b. Clearly, a full width at half-maximum (FWHM) determination is impossible without computer aid.

Table 3. Electron densities of a selection of Martian sites.

\begin{tabular}{|c|c|c|}
\hline Sample & $\begin{array}{l}\text { Electron Density }\left(\times 10^{17} \mathrm{~cm}^{-3}\right) \\
\text { Using the H Line }\end{array}$ & $\begin{array}{l}\text { Electron Density }\left(\times 10^{18} \mathrm{~cm}^{-3}\right) \\
\text { Using the Si Line }\end{array}$ \\
\hline Abbabis & 2.89 & 1.29 \\
\hline Adams_Sound & 2.32 & 0.829 \\
\hline Bald_Rock_Ledge & 2.35 & 1.26 \\
\hline Beech_Mountain_ccam & 2.32 & 0.794 \\
\hline Carrassett & 1.82 & 0.819 \\
\hline Chapeu_Armado & 2.37 & 1.2 \\
\hline Deadman_Pass & 1.86 & 0.81 \\
\hline Dublin_Gulch & 1.85 & 0.899 \\
\hline Emanya & 2.38 & 0.903 \\
\hline Exshaw & 2.35 & 0.826 \\
\hline Finley & 2.39 & 1.23 \\
\hline Furnace_Creek & 2.31 & 0.758 \\
\hline Gold shot & 2.94 & 1.22 \\
\hline Gypsy & 2.31 & 1.16 \\
\hline Hay_Creek_new & 2.32 & 1.23 \\
\hline Humpata & 2.86 & 1.16 \\
\hline Ingraham & 1.85 & 0.782 \\
\hline Iona & 1.32 & 0.885 \\
\hline Jackson_Lake_3 & 2.37 & 0.787 \\
\hline John_Klein_RP3 & 1.84 & 0.769 \\
\hline Kaertok & 2.32 & 0.8 \\
\hline
\end{tabular}


Table 3. Cont.

\begin{tabular}{|c|c|c|}
\hline Sample & $\begin{array}{l}\text { Electron Density }\left(\times 10^{17} \mathrm{~cm}^{-3}\right) \\
\text { Using the } \mathrm{H} \text { Line }\end{array}$ & $\begin{array}{c}\text { Electron Density }\left(\times 10^{18} \mathrm{~cm}^{-3}\right) \\
\text { Using the Si Line }\end{array}$ \\
\hline Kahochella & 1.85 & 0.78 \\
\hline LaValle & 2.39 & 1.22 \\
\hline Loadstone & 1.85 & 1.47 \\
\hline Marent & 1.84 & 1.4 \\
\hline Mukorob & 2.29 & 1.08 \\
\hline Nauaspoort & 2.39 & 1.23 \\
\hline Nullara & 2.32 & 1.26 \\
\hline Oamites & 1.79 & 0.836 \\
\hline Oso_Canyon & 2.32 & 1.26 \\
\hline Palmhorst & 2.31 & 0.764 \\
\hline Pyramid_Hills & 2.36 & 1.33 \\
\hline Quartz_Spring & 1.37 & 0.742 \\
\hline Quechee_Gorge & 1.81 & 1.46 \\
\hline Red_Lodge & 2.32 & 1.5 \\
\hline Ruker_RP_ccam & 1.79 & 0.836 \\
\hline Sawtooth shot & 1.83 & 0.876 \\
\hline Spragueville_ccam & 1.79 & 1.29 \\
\hline Towow & 1.82 & 0.825 \\
\hline Tully & 2.82 & 1.33 \\
\hline Ugab & 2.08 & 1.29 \\
\hline Uutapi & 1.86 & 0.811 \\
\hline Vaqueros_03 & 1.86 & 0.808 \\
\hline Verbrandeberg & 2.37 & 1.32 \\
\hline Wallal & 2.32 & 1.3 \\
\hline Wilhelmstal & 1.85 & 0.779 \\
\hline Yarrada & 2.36 & 1.3 \\
\hline Young_Point & 1.82 & 0.815 \\
\hline Zerrissene & 2.36 & 1.12 \\
\hline Average & $2.17 \times 10^{17}$ & $1.04 \times 10^{18}$ \\
\hline standard deviation & $4.19 \times 10^{16}$ & $2.50 \times 10^{17}$ \\
\hline$\%$ RSD & 19.3 & 24.0 \\
\hline
\end{tabular}

Notes: * For this column, the average, standard deviation, and percent relative standard deviation of all of the electron densities calculated (348 total) using the hydrogen line are reported; ** For this column, the average, standard deviation, and percent relative standard deviation of all of the electron densities calculated (1184 total) using the Si line are reported.

Figure 4 shows a comparison between the average electron densities of the Martian and Earth samples. It is clearly shown that the Earth samples have a significantly lower electron density than the Martian samples. A prior study reported an electron density range of 1.5 to $2.0 \times 10^{17} \mathrm{~cm}^{-3}$ for a synthetic silicate sample using a time delay of $0.5 \mu$ s with a $4.5 \mu$ s gate width over energies ranging from $10 \mathrm{~mJ}$ to $100 \mathrm{~mJ}$ under normal atmospheric conditions on Earth [13]. This current study reports an average of $1.29 \times 10^{17} \mathrm{~cm}^{-3}$; this is close to the lower energy values from the previous study and 
the small difference is most likely due to the time delay and gate width differences. The Martian data showed a larger average electron density using similar energy parameters; it is less than a twofold increase but the results show that the electron densities from the Martian and Earth data are significantly different from each other and that there is more broadening in the hydrogen line with the Martian data.

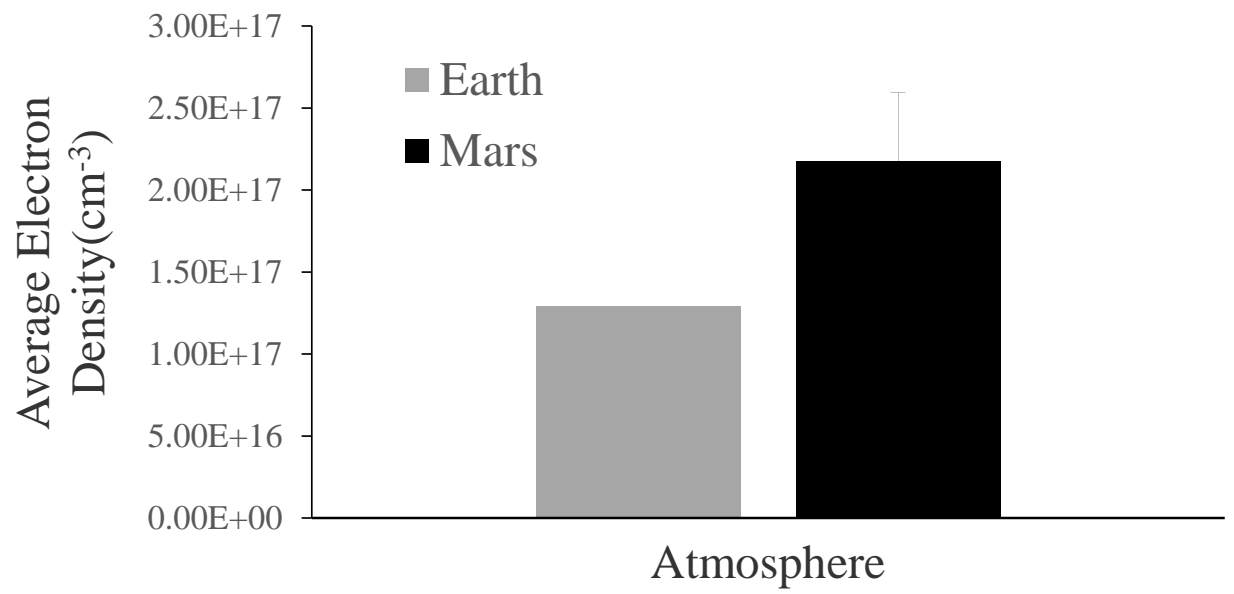

Figure 4. A comparison of the average electron densities using the hydrogen line for Earth and Mars.

The electron density data were also worked up using the Si (I) line at $250.69 \mathrm{~nm}$ due to some discrepancies as noted in the discussion below. All 1184 Martian files were able to be used for electron density determination in this data set. Tables 2 and 3 contain the electron density using the silicon line for the Earth data and Martian data, respectively. These data show a significant decrease in electron density with the Martian data; however, it is less than a twofold decrease. This is further discussed below.

\section{Discussion}

The results in electron density using the hydrogen line from the Martian data tend to contradict what other studies have predicted about the electron density of plasma under Martian conditions. Some research suggests and shows that higher pressures cool the sample the more quickly and increase the Stark broadening, thus increasing the overall electron density. Plasmas formed in lower pressures have a reduction in cooling which decreases the Stark broadening and electron density and allows for increased resolution; this is why the Martian conditions tend to have increased resolution [14-21]. The calculated electron densities in Earth's atmosphere reported here are corroborated with prior results using the same hydrogen line [13]. Electron density higher by a factor of two is predicted by Griem's theory using this hydrogen line [22], but, since the same line and calculation was used for both sets of data (Earth's and Mars's), this impact is not considered here. The Salle paper suggests that lower pressures cause less stark broadening for elements that are found at higher concentrations which have lower energy upper levels that end near the ground state [17]. Their paper also shows that there was no effect on Stark broadening for lower concentration elements with the same types of transitions previously mentioned and for elements at higher concentrations with higher energy upper levels that end in higher energy lower levels [17]. The hydrogen line used here has a higher energy upper level $(12.1 \mathrm{eV})$ and ends in a higher energy lower level (10.2 eV). Both atmospheres (Earth's and Mars's) have trace amounts of hydrogen present. This potentially means that there are other effects, which are causing the increased Stark broadening of the hydrogen line on Mars. A possible explanation for this line broadening increase could be due to elemental concentration effects. There are some studies that suggest that elements found at higher concentrations can produce more ion-electron recombination in the plasma which reduces the overall spectral intensities and causes a wider line profiles leading to increased electron densities $[23,24]$. A reduction in the emission intensities is shown in the Salle 
and Colao papers for the spectral data under a simulated Martian atmosphere. Even though better signal-to-background ratios are calculated for the detected lines in simulated Martian conditions, the overall spectral intensities were decreased under Martian conditions [17,21]. The Colao paper does show reduced electron densities in the $\mathrm{CO}_{2}$ atmosphere due to a narrowing of the neutral silicon line in their spectral data; their electron density was calculated using the neutral silicon line at $250.69 \mathrm{~nm}$. Their samples chosen contained a larger amount of silicon and this silicon line used in their electron density calculation has a lower energy upper level and ends near the ground state; as reported in the Salle paper, the use of this silicon line may be why they saw reduced electron densities under simulated Martian conditions [17,21]. A confirmation of this effect was done by measuring the FWHM of the Si I at $250.69 \mathrm{~nm}$ and calculating in all of the Martian data where the plasma temperatures were calculated (1184 files total) and the Earth data. The FWHM of the Si I line in the Earth data was larger than the FWHM of the Si I line in the Martian data. The average electron density using the Si I emission line was determined to be $1.46 \times 10^{18} \mathrm{~cm}^{-3}$ with a $7.79 \%$ RSD and $1.04 \times 10^{18} \mathrm{~cm}^{-3}$ with a $24 \%$ RSD for the Earth and Mars data, respectively. Both the Earth and Mars data produced much higher electron densities using the silicon line; this was by a factor of approximately 11 and 5 in the Earth and Mars data, respectively. A further examination of different line broadening methods could be explored to help improve the accuracy within the data. Comparing the electron density calculated with the silicon line for the Earth and Mars data, it is clearly shown that the Mars data produced a lower electron density than the Earth data; this shows the pressure, transition, and concentration dependence of the Si I line as noted in the Salle paper [17] while the calculated electron density using the $\mathrm{H}$ line does not have this pressure, transition, and concentration dependence and shows an increase in the electron density with the Martian data.

Furthermore, it should be noted that there are many other LIBS variables other than sample composition and atmosphere which can affect the obtained LIBS spectrum; some of these include laser wavelength, timing parameters, pulse energy, number of laser shots, and the angle of incidence between the laser pulse and sample. This study had no control over the parameters such as target distance, spot size or light collection for the Martian samples, since the rover was controlled by NASA. Even though the samples taken on Earth were made to match some of the rover parameters such as laser energy, other factors such as the varying distance to the target of some samples, could likely affect the Martian data and lead to some variation in the plasma temperature and electron density on those samples $[1,3,8,9]$. Certain trends, especially the presence of a large carbon line and the generally higher plasma temperatures are likely a result of the combined Martian atmosphere and sample composition. Discrepancies in electron density results need a further examination. This could be done by simulating a Martian atmosphere on Earth for a more detailed study to examine this effect.

\section{Material and Methods}

Sample Preparation: The Earth-based samples were synthetic silicate (GBW 07709), red soil (VS 2501-83), black soil (VS 2507-83), and soil (NCS DC73029); these were purchased from Brammer Standards, Houston, TX. These samples were pressed into $35 \mathrm{~mm}$ pellets using a hydraulic press (Carver, Model-C, Wabash, Indiana) to create a smooth surface for LIBS analysis.

LIBS Instrumentation: The Martian data were collected by NASA ChemCam equipment on the Curiosity rover. A description of the ChemCam system is published elsewhere; this contains a brief overview of the system [1-3]. The ChemCam used a Nd:KGW laser that produced $5 \mathrm{~ns}$ pulses at $1067 \mathrm{~nm}$ with energies of up to $14 \mathrm{~mJ}$ reaching the sample. No gate delay was used for the Martian samples as it was unnecessary in Martian conditions. The ChemCam system on the MSL is split into a mast unit and a body unit. The mast unit contains the laser, a telescope, and a remote microimager. The telescope is used to collect the plasma light created by the laser and transmit it into a fiber optic cable that sends the light to three spectrometers. The light is then detected using CCDs (charged coupled devices). The telescope allows the LIBS system to analyze samples up to $7 \mathrm{~m}$ away with 10\% accuracy. The body unit housed the three spectrometers. The spectrometers 
operated in a range from 242-800 nm, excluding 335-385 nm, and 465-510 nm. The spectrometers had a resolution of $0.2 \mathrm{~nm}$. The detectors on these spectrometers were a two-dimensional CCD array. The laser, spectrometer, and CCDs were all synchronized with the laser. The body unit also contained a system for onboard calibration. The ChemCam calibration target assembly (CCCT) contained at least one sample to similar to each of the expected rock types. Each target could accommodate 100 analyses of 75 laser pulses [1-3]. The LIBS Rover took data for each laser shot for each site at each location on that site. The data used here analyzed an accumulation of shot numbers 7 through 16 for location 1 of each site analyzed; shots 1-6 were excluded because those shots were considered to allow the laser to remove the sample area of possible contamination. This data set used here contained over 14,000 data files. There were a total of 1454 sites and ten shots were accumulated for each site. Therefore, 1454 LIBS spectra were analyzed for this part of the project. The data are open access [12], but the data used for this study were downloaded via another website where the files were reformatted to make them easier to use [25].

The Earth data were taken with a laboratory LIBS set-up using a Nd: YAG laser (Surelite II, Continuum, Santa Clara, CA, USA) operating at $1064 \mathrm{~nm}$ using a laser energy of $12 \mathrm{~mJ}$. A $75 \mathrm{~mm}$ focal length lens was used to focus the laser pulse onto the sample. The light was collected by an optical fiber (QP1000-2-UV-VIS, Ocean Optics, Dunedin, FL, USA) placed near the sample. The light was spectrally resolved and detected with an echelle spectrograph (SE200, Catalina, Tucson, AZ, USA) with an ICCD (DH-734-18F-03 IStar, Andor Technology, Belfast, Ireland). The approximate resolving power of the echelle/ICCD is approximately 1700. The laser was operated at $10 \mathrm{~Hz}$. A time delay of $1 \mu \mathrm{s}$ and a $20 \mu$ s gate width were used with a gain of 125 and a three-second exposure; each sample spot represents an accumulation of 30 laser shots. The timing between the laser and detector, the gate-delay, was controlled using a digital delay generator (BNC Model 575-4C Digital Delay and Pulse Generator, Berkeley Nucleonic Corp., San Rafael, CA, USA). Each sample was analyzed five times. The Earth-based LIBS setup is shown in Figure 5.

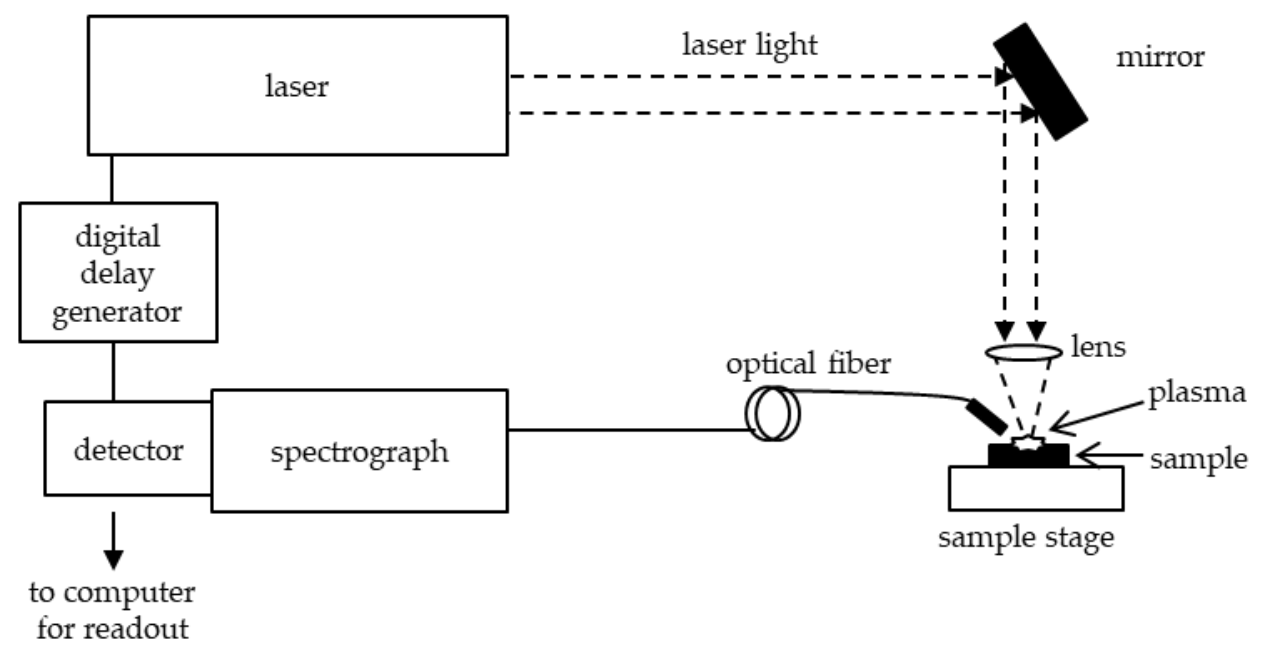

Figure 5. The laser-induced breakdown spectroscopy (LIBS) setup diagram for the Earth-based samples.

Boltzmann plot method: To determine the plasma temperature using the Boltzmann plot method, Equation (1) is used; this gives the spectral line gradient intensity [4,7].

$$
I=\frac{h v A N}{4 \pi}=\frac{h c N_{0} g A}{4 \pi Z} e^{-E_{u / k T}}
$$

In Equation (1), $I$ is the intensity of the transition, $h$ is Planck's constant, $v$ is the frequency, $g$ is the degeneracy, $A$ is the transition probability, $N$ is the absolute number or number density, $c$ is the speed 
of light, $N_{0}$ is the total species population, $\lambda$ is the wavelength, $Z$ is the partition function, $E_{u}$ is the energy of the upper state of emission, $k$ is the Boltzmann constant, and $T$ is the temperature [8].

Rearranging Equation (1) to a linear line format allows for graphical analysis as seen in equation

$$
\ln \left(\frac{I \lambda}{g A}\right)=-\frac{E_{u}}{k T}-\ln \left(\frac{4 \pi Z}{h c N_{0}}\right)
$$

To create the Boltzmann plot, $\ln (I \lambda / g A)$ is plotted against $E_{u}$ in eV. Equation (2) takes the general form of a line, $\mathrm{y}=\mathrm{mx}+\mathrm{b}$ where $\mathrm{x}$ is the $E_{u}, \mathrm{y}$ is the $\ln (I \lambda / g A)$, and the slope, $\mathrm{m}$, is $-1 / k T$. The $E_{u}$, $A$, and $g$ can be found using the National Institute of Standards and Technology (NIST) database; the intensity and wavelength data can be found from the spectra [26].

The Boltzmann plots (Figure 1) were created for the Mars and Earth spectra using a set of titanium lines between 300 and $310 \mathrm{~nm}$. A linear trendline was applied to each of the plots. The temperatures were calculated using the slope of the linear fit [8]. These temperatures were then used to help calculate the electron density of the samples, as explained below. All of the data work up was done in Microsoft Excel.

Electron density method: For calculating the electron densities, the method of using full width at half-maximum (FWHM) with the Stark broadening of spectral lines was used. To determine the electron density of a sample, the FWHM of the hydrogen line at $656.3 \mathrm{~nm}$ line was used; this corresponds to a Balmer alpha line for hydrogen. The linear Stark width for hydrogen lines is shown in Equation (3).

$$
N_{e}=8.02 \times 10^{12}\left(\frac{\Delta \lambda_{1 / 2}}{\alpha_{1 / 2}}\right)^{3 / 2}
$$

where $N_{e}$ is the electron density, $\Delta \lambda_{1 / 2}$ is the FWHM, and $\alpha_{1 / 2}$ is reduced wavelength, which is dependent on both electron density and temperature of the plasma. The values for $\alpha_{1 / 2}$ are provided in Griem's 1974 Appendix IIIa [4,8,9].

The FWHM of the hydrogen was determined for all samples. A Microsoft Excel file was created to calculate the electron density at various temperatures. The FWHM was used to calculate the electron density using the created Excel spreadsheet [8].

On most of the Martian data, a carbon line $(658.8 \mathrm{~nm})$ caused some interferences with the $656.3 \mathrm{~nm}$ hydrogen line; this is shown in Figure 3. Since the peaks tend to be symmetrical in nature, a computer program was created to trace the other portion of the hydrogen and calculate the FWHM of those affected Martian files. The program algorithm was written as follows:

(1) Input the specific wavelength range containing the pertinent peaks;

(2) Find the wavelength corresponding to the carbon peak;

(3) Find the position of the hydrogen peak, which was lower than the carbon peak;

(4) Determine the baseline that is the position corresponding to the minimum density at the left of the carbon peak;

(5) Determine the half-height;

(6) Trace the missing portion of the hydrogen peak using the symmetric method;

(7) Find the position on the electron density curve when the y value of the position is equal to half-height;

(8) Calculate the FWHM.

The program in this paper was run in the Microsoft Windows 1064 bit operating system. The computer was configured with a 16-GB Intel (R) Core (TM) i7-7700 processor. The computer program was implemented in Matlab (R2018a, The Mathworks Inc., Natick, MA, USA). 
The Stark width calculation for the Si line at $250.69 \mathrm{~nm}$ is shown in Equation (4):

$$
\Delta \lambda_{1 / 2} \cong 2 w N_{e} 10^{-16}+3.5 A\left(\frac{N_{e}}{10^{16}}\right)^{1 / 4} \times\left(1-1.2 \times N_{D}^{-1 / 3}\right) w N_{e} 10^{-16}
$$

where $\Delta \lambda_{1 / 2}$ is the FWHM, $w$ is the electron impact half-width, $N_{e}$ is the electron density, $\mathrm{A}$ is the ion-broadening parameter, and $N_{D}$ is the number of particles in the Debye sphere. This equation can be simplified to Equation (5) due to the minimal effect of the second term [27]. Equation (5) was used to calculate the electron density for the Mars and Earth data.

$$
\Delta \lambda_{1 / 2} \cong 2 w N_{e} 10^{-16}
$$

The electron impact half-width for the Mars and Earth data was calculated by plotting the $w$ 's vs. temperature provided by Griem's Plasma Spectroscopy [28] and interpolating the $w$ for the Mars and Earth data based on the calculated temperatures for each spectral file.

A computer program was written to calculate the FWHM of all of the Martian data where temperature results were obtained; this included 1184 files. The program was written (1) to find the position of highest intensity of the silicon peak, (2) to find the position of the minimum intensity in the set wavelength range to determine the baseline, (3) to find the position of the half height between the baseline and silicon peak and (4) to calculate the FWHM distance. The same computer and program were used as noted above for the hydrogen line.

\section{Conclusions}

The Mars and Earth samples produced average temperatures of $18,900 \pm 4640 \mathrm{~K}$ with a $\%$ RSD of $24.5 \%$ and $15,900 \pm 2500 \mathrm{~K}$ with a $\%$ RSD of $15.5 \%$, respectively. For electron density determination using the hydrogen line, the average electron density of the Martian and Earth samples were determined to be $2.17 \times 10^{17} \pm 4.75 \times 10^{16} \mathrm{~cm}^{-3}$ with a \%RSD of $19.3 \%$ and $1.29 \times 10^{17} \pm 1.29 \times 10^{16} \mathrm{~cm}^{-3}$ with a \%RSD of $10.0 \%$, respectively. For electron density determination using the silicon line, the average electron density of the Martian and Earth samples were determined to be $1.04 \times 10^{18} \mathrm{~cm}^{-3}$ with a $24 \%$ RSD and $1.46 \times 10^{18} \mathrm{~cm}^{-3}$ with a $7.79 \%$ RSD, respectively. For both sets of data, there were lower variances for the Earth data; this is most likely due to the smaller sample set and/or the more constant LIBS parameters of the Earth samples. Higher plasma temperatures were obtained with the Mars data, though they were not significantly different from each other. Depending on the line chosen for electron density analysis, either an increase or decrease in electron density was found in the Martian data; further work would need to be done to determine why these differences were found and which is a better representation of the electron density at reduced pressures.

Author Contributions: R.C.C. designed the project and the experiment and assisted with the data collection for the Earth samples and data analysis for all of the data, J.S. collected the Earth data and performed the data analysis on both Earth and Martian data, and S.T. designed the computer program that calculated the FWHM of the hydrogen line on the Martian spectra that showed the carbon interference, and also wrote a program to determine the FWHM of the Si line for the Martian data. All authors have read and agreed to the published version of the manuscript.

Funding: This work was funded by Alvernia University's student undergraduate research fellow (SURF) program and Alvernia's Math and Science department.

Acknowledgments: Special thanks to M.D. Dyar from the Department of Astronomy at Mount Holyoke College, who provided access to the reformatted ChemCam LIBS data. The authors would also like to acknowledge C. Parigger for his beneficial discussion on electron density.

Conflicts of Interest: The authors declare no conflict of interest. 


\section{References}

1. Wiens, R.C.; Maurice, S.; Lasue, J.; Forni, O.; Anderson, R.B.; Clegg, S.; Bender, S.; Blaney, D.; Barraclough, B.L.; Cousin, A.; et al. Pre-flight calibration and initial data processing for the ChemCam laser-induced breakdown spectroscopy instrument on the Mars Science Laboratory rover. Spectrochim. Acta Part B 2013, 82, 1-27. [CrossRef]

2. Wiens, R.C.; Maurice, S.; Barraclough, B.; Sauccoccio, M.; Barkley, W.C.; Bell, J.F.; Bender, S.; Bernardin, J.; Blaney, D.; Blank, J.; et al. The ChemCam Instrument Suite on the Mars Science Laboratory (MSL) Rover: Body Unit and Combined Systems Tests. Space Sci. Rev. 2012, 170, 167-227. [CrossRef]

3. Maurice, S.; Wiens, R.C.; Saccoccio, M.; Barraclough, B.; Gasnault, O.; Forni, O.; Mangold, N.; Baratoux, D.; Bender, S.; Berger, G.; et al. The Chem Cam Insrument Suite on the Mars Science Laboratory (MSL) Rover: Science Objectives and Mast Unit Description. Space Sci. Rev. 2012, 170, 96-166. [CrossRef]

4. Cremers, D.A.; Radziemski, L.J. Handbook of Laser-Induced Breakdown Spectroscopy; John Wiley \& Sons, Ltd.: West Sussex, UK, 2006.

5. Hahn, D.W.; Omenetto, N. Laser-Induced Breakdown Spectroscpy (LIBS), Part I: Review of Basic Diagnosics and Plasma-Particle Interactions: Still-Challenging Issues within the Analytical Plasma Community. Appl. Specstrosc. 2010, 64, 335A-366A. [CrossRef] [PubMed]

6. Harilal, S.S.; Bindhu, C.V.; Issac, R.C.; Nampoori, V.P.N.; Vallabhan, C.P.G. Electron density and temperature measurements in a laser produced carbon plasma. J. Appl. Phys. 1997, 82, 2140-2146. [CrossRef]

7. Unnikrishnan, V.K.; Alti, K.; Kartha, V.B.; Santhosh, C.; Gupta, G.P.; Suri, B.M. Measurements of plasma temperature and electon density in laser-induced copper plasma by time-resolved spectroscopy of neutral atom and ion emissions. Pranmana J. Phys. 2010, 74, 983-993. [CrossRef]

8. Najarian, M.L.; Chinni, R.C. Temperature and Electron Density Determination on Laser-Induced Breakdown Spectroscopy (LIBS) Plasmas: A Physical Chemistry Experiment. J. Chem. Educ. 2013, 90, 244-247. [CrossRef]

9. Griems, H.R. Spectral Line Broadening by Plasmas; Academic Press: New York, NY, USA, 1974.

10. Griems, H.R.; Halenka, J.; Olchawa, W. Comparison of hydrogen Balmer-alpha Stark profiles measured at high electron densities with theoretical results. J. Phys. B At. Mol. Opt. Phys. 2005, 38, 975-1000. [CrossRef]

11. Ashkenazy, J.; Kipper, R.; Caner, M. Spectroscopic Measurements of Electron Density of Capillary Plasma Based on Stark Broadening of Hydrogen Lines. Phys. Rev. A 1991, 43, 5568-5574. [CrossRef]

12. NASA Website, PDS Geosciences Node, Washington University in St. Louis. Available online: https://pds-geosciences.wustl.edu/ (accessed on 12 June 2020).

13. Kurek, L.; Najarian, M.L.; Cremers, D.A.; Chinni, R.C. Dependence of Laser-Induced Breakdown Spectroscopy Results on Pulse Energies and Timing Parameters Using Soil Simulants. J. Vis. Exp. 2013, 79, e50876. [CrossRef]

14. Effenberger, A.J.; Scott, J.R. Effect of Atmospheric Conditions on LIBS Spectra. Sensors 2010, 10, 4907-4925. [CrossRef] [PubMed]

15. Vadillo, J.M.; Fernandez Romero, J.M.; Rodriguez, C.; Laserna, J.J. Depth-resolved Analysis by Laser-induced Breakdown Spectrometry at Reduced Pressure. Surf. Interface Anal. 1998, 26, 995-1000. [CrossRef]

16. Vadillo, J.M.; Fernandez Romero, J.M.; Rodriguez, C.; Laserna, J.J. Effect of Plasma Shielding on Laser Ablation Rate of Pure Metals at Reduced Pressure. Surf. Interface Anal. 1999, 27, 1009-1015. [CrossRef]

17. Salle, B.; Cremers, D.A.; Maurice, S.; Wiens, R.C. Laser-induced breakdown spectroscopy for space exploration applications: Influence of the ambient pressure on the calibration curves prepared from soil and clay samples. Spectrochim. Acta Part B 2005, 60, 479-490. [CrossRef]

18. Brennetot, R.; Lacour, J.L.; Vors, E.; Rivoallan, A.; Vailhen, D.; Maurice, S. Mars Analysis by Laser-Induced Breakdown Spectroscopy (MALIS): Influence of Mars Atmosphere on Plasma Emission and Study of Factors Influencing Plasma Emission with the use of Doehlert Designs. Appl. Spec. 2003, 57, 744-752. [CrossRef]

19. Aguilera, J.A.; Aragon, C. A comparison of the temperatures and electron densities of laser-produced plasmas obtained in air, argon, and helium at atmospheric pressure. Appl. Phys. A 1999, 69, S475-S478. [CrossRef]

20. Dreyer, C.B.; Mungas, G.S.; Thanh, P.; Radziszewski, J.G. Study of sub-mJ-excited laser-induced plasma combined with Raman spectroscopy under Mars atmosphere-simulated conditions. Spectrochim. Acta Part B 2007, 62, 1448-1459. [CrossRef]

21. Colao, F.; Fantoni, R.; Lazic, V.; Paolini, A. LIBS application for analyses of martian crust analogues: Search for the optimal experimental parameters in air and $\mathrm{CO}_{2}$ atmosphere. Appl. Phys. A 2004, 79, 143-152. [CrossRef] 
22. Parigger, C.G.; Plemmons, D.H.; Oks, E. Balmer series $\mathrm{H}_{\beta}$ measurements in a laser-induced hydrogen plasma. Appl. Opt. 2003, 42, 5992-6000. [CrossRef]

23. Wisbrun, R.; Schechter, I.; Niessner, R.; Schrfder, H.; Kompa, K. Detector for trace elemental analysis of solid environmental samples by laser plasma spectroscopy. Anal. Chem. 1994, 66, 2964-2975. [CrossRef]

24. Eppler, A.S.; Cremers, D.A.; Hickmott, D.D.; Ferris, M.J.; Koskelo, A.C. Matrix effects in the detection of Pb and $\mathrm{Ba}$ in soils using laser-induced breakdown spectroscopy. Appl. Spec. 1996, 50, 1175-1181. [CrossRef]

25. DEVAS, Data Exploration, Visualization, and Analysis for Spectroscopy Website. Available online: Nemo.mtholyoke.edu (accessed on 12 June 2020).

26. National Institute of Standards and Technology (NIST), Physics Measurements Laboratory, NIST Atomic Spectra Database Lines Form Website. Available online: https://physics.nist.gov/PhysRefData/ASD/lines_ form.html (accessed on 10 December 2019).

27. Milan, M.; Laserna, J.J. Diagnostics of silicon plasmas produced by visible nanosecond laser ablation. Spectrochim. Acta Part B 2001, 56, 275-288. [CrossRef]

28. Griems, H.R. Plasma Spectroscopy; McGraw Hill Book Company: New York, NY, USA, 1964.

(C) 2020 by the authors. Licensee MDPI, Basel, Switzerland. This article is an open access article distributed under the terms and conditions of the Creative Commons Attribution (CC BY) license (http://creativecommons.org/licenses/by/4.0/). 